\title{
Durvalumab-induzierte Myokarditis, Myositis und Myasthenia gravis: ein Fallbericht
}

\author{
Jason Cham Daniel Ng Laura Nicholson \\ Department of Internal Medicine, Scripps Clinic/Scripps Green Hospital, La Jolla, CA, USA
}

\section{Schlüsselwörter}

Checkpoint-Inhibitoren · Immuntherapie · Nebenwirkungen .

Myokarditis · Myositis · Myasthenia gravis · nichtkleinzelliger

Lungenkrebs

\section{Zusammenfassung}

Hintergrund: Immuncheckpoint-Inhibitoren sind wirksame Therapien für ein breites Spektrum von bösartigen Erkrankungen. Ihre zunehmende Verbreitung hat zu einer Vielzahl von immunbedingten Nebenwirkungen geführt, unter anderem zu Haut-, MagenDarm-, Lungen-, endokrinen, kardialen und neurologischen Komplikationen.

Fallbericht: Wir stellen den Fall eines 72-jährigen weißen Mannes mit nichtkleinzelligem Lungenkrebs vor, der nach 2 Zyklen Durvalumab wegen Dyspnoe aufgenommen wurde. Bei ihm wurden signifikant erhöhte Kreatinin-Kinase- und Troponin-Werte im Serum festgestellt, die Herzkatheteruntersuchung war negativ. Während seines Krankenhausaufenthalts entwickelte er eine fortschreitende Dyspnoe und eine neu aufgetretene axiale Schwäche, was schließ- lich zur Diagnose einer Durvalumab-induzierten Myokarditis, Myasthenia gravis und Myositis führte.

Schlussfolgerungen: Soweit uns bekannt ist, handelt es sich hierbei um den ersten Fall einer Kombination aus Myokarditis, Myasthenia gravis und Myositis, die durch den Antikörper gegen PDL1 (programmed cell death ligand 1) ausgelöst wurde. Der Einsatz immunologischer Wirkstoffe hat zwar insgesamt zu besseren Behandlungsergebnissen bei Krebs geführt, aber auch zu einem breiten Spektrum immunbedingter Nebenwirkungen. Wir geben einen Überblick über die Diagnostik und Behandlung von Patienten mit diesen immunbedingten Nebenwirkungen und betonen, wie wichtig eine frühzeitige Erkennung ist, da es zu einer schnellen Verschlechterung kommen kann.

\section{Hintergrund}

Durvalumab (MedImmune/AstraZeneca) ist ein humaner monoklonaler Immunglobulin-G1-kappa-Antikörper, der gegen PD-L1 (programmed cell death ligand 1) gerichtet ist. Er verfügt nachweislich über eine signifikante Antitumoraktivität und ist von der US Food and Drug Administration (FDA) für die Behandlung von nichtkleinzelligem Lungenkrebs und Urothelkarzinomen zugelassen. Im März 2020 kam die Zulassung von Durvalumab in Kombination mit Etoposid und entweder Carboplatin oder Cisplatin als Erstlinienbehandlung von Patienten mit ausgedehntem kleinzelligem Lungenkrebs hinzu [1]. Immuncheckpoint-Inhibi- toren (ICI) sind bei der Behandlung zahlreicher bösartiger Tumoren heute eine tragende Säule; sie stehen jedoch auch im Zusammenhang mit immunbedingten unerwünschten Wirkungen (irAE). Die häufigsten irAE für Durvalumab sind immunvermittelte dermatologische Reaktionen (1,6\%), Colitis (1,6\%), Hepatitis (1\%), Nephritis (0,3\%) und Endokrinopathien einschließlich Hypothyreose $(7,3 \%)$ und Hyperthyreose $(1,4 \%)$, Nebenniereninsuffizienz $(0,4 \%)$, Diabetes mellitus Typ $1(<0,1 \%)$ und Hypopituitarismus/Hypophysitis $(<0,1 \%)[1]$. Hier stellen wir einen Patienten vor, der eine seltene Kombination von unerwünschten Wirkungen entwickelte: Myokarditis, Myositis und Myasthenia gravis (MG) als Folge der PD-L1-Inhibition mit Durvalumab. 


\section{Fallbericht}

Bei einem 72-jährigen weißen Mann mit einer Vorgeschichte von Prostatakrebs, zentraler seröser Retinopathie, obstruktiver Schlafapnoe, Diabetes mellitus Typ 2, Bluthochdruck und leichter chronisch obstruktiver Lungenerkrankung, aber ohne bekannte koronare Herzkrankheit oder neurologische Störungen in der Vorgeschichte, wurde 2013 erstmals ein nichtkleinzelliges Lungenkarzinom (NSCLC) im linken Unterlappen diagnostiziert, das noch im selben Jahr im Rahmen einer Lobektomie des linken Unterlappens entfernt wurde. Bei einer Bildgebung im Jahr 2014 wurde bei ihm ein Knoten im rechten Unterlappen festgestellt, und es wurde ein zweites primäres NSCLC diagnostiziert, woraufhin er sich einer stereotaktischen Bestrahlung (SBRT) unterzog. Im Jahr 2017 wurde bei ihm ein vergrößerter Knoten im linken Oberlappen festgestellt, der mit SBRT behandelt wurde. Eine Computertomografie (CT) im September 2018 war wegen eines größer werdenden Knotens im rechten Oberlappen mit Beteiligung des rechten paratrachealen Lymphknotens auffällig. Im anschließenden Positronen-Emissions-Tomografie (PET)-CT-Scan wurden mehrere Fluorodeoxyglukose (FDG)-avide Mediastinalund rechte Hilusknoten festgestellt, die auf ein Malignom hinweisen. Ein metastatisches bronchogenes Adenokarzinom wurde durch endobronchialen Ultraschall mit transbronchialer $\mathrm{Na}$ delaspiration bestätigt, wobei die Pathologie 5/5 positive Lymphknoten ergab. Die immunhistochemische Untersuchung des Lymphknotens auf PD-L1 mit 22C3 (Keytruda) ergab eine hohe PD-L1-Expression mit 80\% Tumoranteil. Die Magnetresonanztomografie (MRT)-Untersuchung des Gehirns ergab keinen Hinweis auf eine intrakranielle metastatische Erkrankung. Bei ihm wurde ein Adenokarzinom im Stadium IIIB (TxN3M0) diagnostiziert und er wurde mit 6 Zyklen wöchentlichem Cisplatin und SBRT behandelt. Anschließend wurde bei ihm eine adjuvante Erhaltungstherapie mit Durvalumab begonnen, wobei er 2 Zyklen $(10 \mathrm{mg} / \mathrm{kg})$ an den Behandlungstagen 0 und 15 erhielt. Unser Patient kam am 18. Tag in die Notaufnahme, als er bereits 4 Tage an Kurzatmigkeit, Schwäche und einem Druckgefühl in der Brust litt. Bei der Aufnahmeuntersuchung zeigten sich signifikante seitliche ST-Hebungen und ein anfänglicher Troponinwert von 7,1 ng/dl. Er unterzog sich umgehend einer Linksherzkatheteruntersuchung, die ein negatives Ergebnis für eine obstruktive koronare Herzerkrankung ergab. Das transthorakale Echokardiogramm ergab eine normale Ejektionsfraktion mit leichter linksventrikulärer konzentrischer Hypertrophie.

Die Aufnahmediagnose lautete Myoperikarditis unklarer Ätiologie. Am 4. Krankenhaustag wurde bei ihm ein anhaltend erhöhter Troponinspiegel im Blutserum festgestellt, der einen Spitzenwert von $12 \mathrm{ng} / \mathrm{ml}$ erreichte. Sein Kreatinin-Kinase (CK)-Level im Serum war ebenfalls erhöht und erreichte einen Spitzenwert von 9262 U/l. Er hatte einen anhaltenden leichten hepatozellulären Transaminaseanstieg (Spitzenwert Aspartat-Aminotransferase (AST) 72, Alanin-Aminotransferase (ALT) 531). Ein Einsatz von Kortikosteroiden wurde mit dem Patienten wegen der Gefahr von irAE besprochen; der Patient lehnte dies jedoch aufgrund seiner zentralen serösen Retinopathie ab. Bei der Aufnahme wurde bei ihm eine Dysphagie mit Regurgitation von fester und flüssiger Nahrung festgestellt. Bei einer Untersuchung durch Bariumbreischluck und Ösophagogastroduodenoskopie am dritten Krankenhaustag wurden keine strukturellen Anomalien festgestellt. Am 9. Krankenhaustag entwickelte der Patient eine fortschreitende axiale Schwäche mit zunehmenden Schwierigkeiten, seinen Kopf im Sitzen aufrecht zu halten. Es wurde ein Neurologe konsultiert, der den starken Verdacht einer myasthenischen Krise hatte, die dann durch eine verminderte negative Inspirationskraft und durch erhöhte Acetylcholinrezeptor (AchR) bindende und blockierende Antikörper bestätigt wurde. Aufgrund seines sich verschlechternden Atemstatus wurde er auf die Intensivstation verlegt und noch am selben Tag zur Sicherung der Atemwege intubiert. Die am 10. Krankenhaustag durchgeführte MRT-Untersuchung des Gehirns war wegen 12 neuer metastatischer Läsionen mit umgebendem vasogenem Ödem auffällig. Der Patient erhielt hochdosierte Kortikosteroide in einer Dosierung von $1 \mathrm{mg} / \mathrm{kg} /$ Tag und unterzog sich am 10. Krankenhaustag einer Plasmapherese, die 5-mal durchgeführt wurde. Leider konnte er nicht von der mechanischen Beatmung entwöhnt werden und benötigte ein Tracheostoma sowie eine perkutane endoskopische Gastrostomie.

Nach mehreren Gesprächen über die Behandlungsziele wurde der Patient am 36. Krankenhaustag von der Intensivstation in eine Langzeit-Akutpflegeeinrichtung verlegt, da er auf mechanische Beatmung angewiesen war. Die Diagnosen Myoperikarditis, Myositis und myasthenische Krise wurden auf eine immunvermittelte Reaktion auf Durvalumab zurückgeführt.

\section{Diskussion und Schlussfolgerungen}

Die Differenzialdiagnose für Dyspnoe und Schwäche ist sehr umfassend, aber bei einem Patienten, der mit einer Immuntherapie behandelt wird, können sie die Erstsymptome eines irAE sein. In diesem Bericht wird ein Patient vorgestellt, bei dem sich eine seltene Kombination von unerwünschten Wirkungen entwickelte: Myokarditis, Myositis und MG als Folge der PD-L1-Hemmung bei Durvalumab.

Eine Myokarditis im Zusammenhang mit ICI ist ein seltenes irAE mit einer berichteten Inzidenz von $0,04-1,14 \%$, geht jedoch mit einer Mortalität von 25-50\% einher [2]. Bei weiblichen Patienten, Patienten im Alter von über 75 Jahren und Patienten, bei denen gleichzeitig andere ICI eingesetzt werden, wurde ein deutlich erhöhtes Risiko für eine Myokarditis festgestellt [3]. Es gibt ein breites Spektrum an Schweregraden, das von einer asymptomatischen Erhöhung der Herzenzymwerte bis hin zu Endorganstörungen reicht, wie sie von der American Society of Clinical Oncology kategorisiert werden. Dieser Patient wies eine ST-Hebung und deutlich erhöhte Herzenzymwerte auf, hatte aber keine obstruktive koronare Herzkrankheit, was zur Diagnose einer wahrscheinlich ICI-bedingten Myokarditis führte. Die Erstbehandlung besteht aus hochdosierten Kortikosteroiden und dem sofortigen Absetzen des schädigenden Wirkstoffs. Die Möglich-
38

Kompass Autoimmun 2022;4:37-39 DOI: $10.1159 / 000521937$ 
keiten der Zweitlinienbehandlung sind noch nicht bewiesen, beinhalten aber intravenöses Immunglobulin, Mycophenolat, Infliximab, Alemtuzumab und Abatacept [4]. Unser Patient hatte aufgrund seiner zentralen serösen Retinopathie eine relative Kontraindikation für Steroide, wurde aber angesichts seiner schweren Symptome schließlich mit hochdosierten Steroiden und Plasmapherese behandelt.

Paraneoplastische neurologische Syndrome wie das Lambert-Eaton-Myasthenie-Syndrom (LEMS) sind eindeutig mit dem Lungenkarzinom in Verbindung gebracht worden. Beim Auftreten von Atemversagen wurde LEMS vermutet; der Patient wies jedoch negative Antikörper gegen spannungsabhängige Kalziumkanäle auf, was nicht mit der Diagnose vereinbar ist. MG als paraneoplastisches Syndrom bei primären Lungenkarzinomen ist in der Literatur nicht eindeutig beschrieben worden; daher wurde angenommen, dass die myasthenische Krise unseres Patienten durch die ICI verursacht wurde [5]. Die ICI-bedingte MG ist ein seltenes irAE mit einer gemeldeten Inzidenz von weniger als $1 \%$. Eine ICIbedingte MG tritt häufig zwischen 2 und 6 Wochen nach Beginn der Behandlung mit Checkpoint-Inhibitoren auf. Angesichts des zeitlichen Verlaufs der Symptome ist die ICI-bedingte MG also die wahrscheinlichere Diagnose. Im Vergleich zur klassischen MG, die eine bimodale Altersverteilung aufweist, die bei Frauen überwiegt, scheint die mit ICI assoziierte MG hauptsächlich bei Männern aufzutreten, und zwar mit einem vergleichsweise späteren Krankheitsbeginn [6]. 2 pathogene Autoantikörper sind bei der Mehrheit der MG-Patienten vorhanden: 85\% haben AntiAchR-Autoantikörper und 8\% haben Autoantikörper gegen die Muskel-spezifische Tyrosinkinase (MuSK) [7]. Bei unserem Patienten wurden Anti-AchR-Antikörper festgestellt. In einer großen retrospektiven Studie zur immuntherapeutisch induzierten MG durch den Anti-PD-1-Wirkstoff Nivolumab entwickelten 8 von 12 Patienten schwere Symptome einschließlich einer myasthenischen Krise [8]. Die MG trat früh im Behandlungsverlauf auf (innerhalb von 1 oder 2 Behandlungen) und schritt bei den Patienten schnell fort, was mit dem klinischen Verlauf unseres Patienten übereinstimmt. Diese seltene Nebenwirkung muss umgehend erkannt werden, da angesichts des Potenzials eines akuten Atemversagens infolge einer fortschreitenden MG eine Unterstützung der Atmung entweder durch nichtinvasive Überdruckbeatmung oder mechanische Beatmung erforderlich sein kann. Bei Patienten mit leichten Symptomen kann Pyridostigmin eingesetzt werden, aber bei Patienten mit schweren Symptomen und schnellem Fortschreiten ist eine Immunsuppression mit intravenösem Methylprednisolon erforderlich [4]. Es besteht eine gewisse Evidenz, dass intravenöses Immunglobulin (IVIG) und Plasmaaustausch als Erstlinientherapie im Vergleich zur alleinigen Verabreichung von Steroiden bessere Ergebnisse erzielen könnten [9].

Schließlich kann bei Patienten, die eine MG entwickeln, auch eine Myositis auftreten. In den meisten gemeldeten Fällen war die CK deutlich erhöht, wobei die gemeldeten Werte zwischen 1200 und 8729 IU/l lagen [10]. Obwohl bei unserem Patienten und in den meisten berichteten Fällen keine Muskelbiopsie durchgeführt wurde, spiegelt das Vorhandensein eines erhöhten CK-Spiegels zusammen mit den Transaminasen wahrscheinlich eher eine Muskelschädigung als eine Leberschädigung wider, da unser Patient normale Bilirubin- und INR-Werte (INR = international normalized ratio) aufwies und seine Leberbildgebung unauffällig war. In einer retrospektiven Beobachtungsstudie an Patienten mit idiopathischer MG und entzündlichen Myopathien wiesen 7 von 8 Patienten antistriationale Autoantikörper auf, und 3 dieser Patienten entwickelten auch eine Myokarditis [11]. Dies deutet darauf hin, dass es Autoimmunziele mit ähnlichen Epitopen auf Herz- und Skelettmuskeln geben könnte. ICI-induzierte MG mit antiretroviralen Antikörpern wird mit einem raschen Fortschreiten und schwereren Verläufen in Verbindung gebracht, sodass ein frühzeitiges Antikörper-Screening zur frühzeitigen Erkennung und Prognose schwerer irAE beitragen kann [11].

Die Kombination von Myokarditis, MG und Myositis wurde bei verschiedenen ICI, einschließlich Ipilimumab und Nivolumab, berichtet [12]. Über eine Myokarditis wurde schon früher bei Patienten berichtet, die mit Durvalumab behandelt wurden, aber dies ist unseres Wissens der erste Fallbericht einer Anti-PD-L1induzierten Kombination von Myokarditis, MG und Myositis [13]. Zwar wird den ICI ein besseres Verträglichkeitsprofil als der Chemotherapie nachgesagt, doch ist es wichtig, die große Bandbreite an unterschiedlichen Gewebearten zu berücksichtigen, die von immunvermittelten Entzündungen betroffen sein können, was zu lebensbedrohlichem Organversagen führen kann. irAE treten am häufigsten im endokrinen System, in der Haut, in der Lunge und im Magen-Darm-Trakt auf. Die behandelnden Ärzte müssen jedoch auf weniger häufige Immundiathesen achten, darunter neuropathische und myopathische Syndrome wie die Trias aus MG, Myokarditis und Myositis, an der unser Patient gelitten hat. Hier haben wir die Grundsätze der Diagnose und Behandlung dieser seltenen, aber potenziell tödlichen Komplikationen erläutert.

\section{Zustimmung zur Veröffentlichung}

Eine schriftliche Einverständniserklärung wurde vor Veröffentlichung des Fallberichts vom Patienten/von der Ehefrau des Patienten eingeholt. Eine Kopie der schriftlichen Einverständniserklärung zur Prüfung liegt dem Editor-in-Chief des vor.

\section{Interessenskonflikte}

Keine genannt. Alle Autoren erklärten, dass keine finanzielle Interessenskonflikte in Bezug zur vorliegenden Arbeit bestehen.

\section{Lizenzangabe}

Cham J, Ng D, Nicholson L: Durvalumab-induced myocarditis, myositis, and myasthenia gravis: a case report. J Med Case Rep. 2021;15(1):278. (DOI: 10.1186/s13256-021-02858-7). ${ }^{\circledR} 2021$ The Author(s). (Übersetzung; Abbreviations, Acknowledgements, Author's contributions, Funding, Availability of data and materials, Ethics approvel and consent to participate und Publisher's Note gekürzt), lizensiert unter CC BY 4.0 (https://creativecommons.org/licenses/by/4.0/deed.de).

\section{Literatur}

Die Literatur ist unter www.karger.com/doi/10.1159/000521937 abrufbar. 